\title{
Computation of Lie supersymmetries for the supersymmetric two bosons equations
}

\author{
M. A. Ayari* $\quad$ M. I. Ayari ${ }^{\dagger} \quad$ V. Hussin ${ }^{\ddagger}$
}

CRM-2552

July 1998

${ }^{*}$ Centre de Recherches Mathématiques, Université de Montréal C.P. 6128, Succ. Centre-Ville Montréal (Qc), H3C 3J7 Canada

${ }^{\dagger}$ Centre de Recherches Mathématiques, Université de Montréal C.P. 6128, Succ. Centre-Ville Montréal (Qc), H3C 3J7 Canada

${ }_{\ddagger}^{\ddagger}$ Centre de Recherches Mathématiques, Université de Montréal C.P. 6128, Succ. Centre-Ville Montréal (Qc), H3C 3J7 Canada 



\begin{abstract}
Working in a superspace, we compute the Lie-point symmetries for the supersymmetric two bosons equations. Computer algebra has helped us to skip the tedious calculations. Translational symmetry supergroups are used to reduce the supersymmetric two bosons equations to an ordinary differential supersystem which involves two even and two odd dependent variables. Some explicit solutions are presented.
\end{abstract}

PACS NUMBERS: 02.20.Sv, 02.30.Jr, 11.30.Pb.

\title{
Résumé
}

Dans le superespace, nous calculons le groupe de Lie des symétries pour les équations à deux bosons supersymétriques. Le programme GLie écrit dans le langage MAPLE nous permet d'écrire facilement les superéquations déterminantes qui conduisent aux générateurs de la superalgèbre correspondante. On impose l'invariance par translations des solutions pour réduire le supersytéme à un supersystème contenant une seule variable indépendante. Ce dernier se décompose en quatre équations contenant deux variables dépendantes paires et deux impaires. Finalement, on présente quelques solutions explicites. 



\section{Introduction}

For the past decade we have seen in the mathematical-physics area an increasing interest for a new kind of equations called systems of Grassmann-valued differential equations (SGVDE) or superequations. Such ones handle both even (commuting or bosonic) and odd (anticommuting or fermionic) variables. The study of integrability and conservation laws of these superequations were the subject of many papers [1]-[9] where in most cases they have succeeded in presenting Lax pairs and conservation laws. Due to the complexity of such superequations, it is often difficult to give explicit solutions. Besides there is no theory or explicit algorithms on how to solve them as in the classical case. Different attempts were made to give explicit solutions using methods such as the Cartan-Kähler integration theorem [10] for first-order superequations.

In this paper, the calculation technique of Lie-point symmetry supergroups of SGVDE [11] is used to give some solutions of the supersymmetric two bosons equations. This method has already helped us in finding some explicit solutions in terms of Jacobi elliptic and hypergeometric functions for the supersymmetric Korteweg-de-Vries equation $(N=2)$ [12]-[13].

The contents of this paper is as follows: in Section 2, a general SGVDE is given in terms of Grassmann-valued independent and dependent variables. The algorithm for determining all symmetries of this system is described as a simple generalisation of the one given in the books of Olver [14] and Bluman [15]. The supersymmetric two bosons (TB) equations are presented in section 3. The computation of Lie superalgebras for such supersymmetric equations is given in section 4 . Translationally invariant solutions are featured in section 5. Finally, we present in an appendix the input and output files of the computation that have led to the determining superequations.

\section{Algorithm for symmetry superalgebra of SGVDE}

The framework to study such SGVDE calls for the concept and notions of superspace, supermanifolds and superdifferential geometry. For an extensive development of this formalism we refer the reader to Berezin [16] and Kostant [17]. Here we just give the major equations of the extension of Lie's technique for SGVDE [11].

Let us consider the general case of a nonlinear SGVDE of $s$ equations of order $k=\left(k_{1} ; k_{2}\right)$ denoted by

$$
\Delta_{\nu}\left(\mathrm{X}, \Theta, \mathrm{A}^{\left(k_{1}\right)}, \mathrm{Q}^{\left(k_{2}\right)}\right)=0, \quad \nu=1, \ldots, s,
$$

with $m$ independent even variables $\mathrm{X}=\left\{x_{1}, \ldots, x_{m}\right\}, n$ independent odd variables $\Theta=\left\{\theta_{1}, \ldots, \theta_{n}\right\}, q$ dependent even variables $\mathrm{A}=\left\{A^{1}, A^{2}, \ldots, A^{q}\right\}$ and $p$ dependent odd variables $\mathrm{Q}=\left\{Q^{1}, Q^{2}, \ldots, Q^{p}\right\}$.

Let us recall that the following properties are satisfied for arbitrary odd variables $\eta_{i}$ : we have $\eta_{i} \eta_{j}=-\eta_{j} \eta_{i}$ and $\eta_{i}^{2}=0,1 \leq i, j \leq r$.

In the spirit of Lie, a one parameter- $\epsilon$ local point transformation has the form

$$
\begin{array}{ll}
\mathrm{X}^{\prime}=\Omega_{\epsilon}^{1}(\mathrm{X}, \Theta, \mathrm{A}, \mathrm{Q}), & \Theta^{\prime}=\Omega_{\epsilon}^{2}(\mathrm{X}, \Theta, \mathrm{A}, \mathrm{Q}), \\
\mathrm{A}^{\prime}=\Omega_{\epsilon}^{3}(\mathrm{X}, \Theta, \mathrm{A}, \mathrm{Q}), & \mathrm{Q}^{\prime}=\Omega_{\epsilon}^{4}(\mathrm{X}, \Theta, \mathrm{A}, \mathrm{Q}),
\end{array}
$$

where $\epsilon$ is an homogeneous Grassmann variable and denotes the supergroup parameter. The supervector valued functions $\Omega_{\epsilon}^{i}, i=1, \ldots, 4$ depend only on the variables $\mathrm{X}, \Theta, \mathrm{A}$ and $\mathrm{Q}$ (not on the derivatives of $\mathbf{A}$ and $\mathbf{Q}$ ). So the symmetry supergroup $H$ of a SGVDE is the larger supergroup of transformations (2) leaving (1) invariant. 
Expanding each relation in (2) around the identity $\epsilon=0$, one can generate the following infinitesimal transformations

$$
\begin{aligned}
x_{i}^{\prime} & =x_{i}+\epsilon \Xi^{i}(\mathrm{X}, \Theta, \mathrm{A}, \mathrm{Q})+O\left(\epsilon^{2}\right), \quad i=1, \ldots, m, \\
\theta_{j}^{\prime} & =\theta_{j}+\epsilon \Gamma^{j}(\mathrm{X}, \Theta, \mathrm{A}, \mathrm{Q})+O\left(\epsilon^{2}\right), \quad j=1, \ldots, n, \\
A^{\prime r} & =A^{r}+\epsilon \Phi^{r}(\mathrm{X}, \Theta, \mathrm{A}, \mathrm{Q})+O\left(\epsilon^{2}\right), \quad r=1, \ldots, q, \\
Q^{\prime l} & =Q^{l}+\epsilon \Lambda^{l}(\mathrm{X}, \Theta, \mathrm{A}, \mathrm{Q})+O\left(\epsilon^{2}\right), \quad l=1, \ldots, p,
\end{aligned}
$$

where the functions $\Xi^{i}, \Gamma^{j}, \Phi^{r}$ and $\Lambda^{l}$ are the infinitesimals of the transformations for the independent and dependent (even and odd) variables. In consequence, a basis for the corresponding symmetry superalgebra $H$ will be obtained by considering

$$
\mathrm{V}=\sum_{i=1}^{m} \Xi^{i} \frac{\partial}{\partial x_{i}}+\sum_{j=1}^{n} \Gamma^{j} \frac{\partial}{\partial \theta_{j}}+\sum_{r=1}^{q} \Phi^{r} \frac{\partial}{\partial A^{r}}+\sum_{l=1}^{p} \Lambda^{l} \frac{\partial}{\partial Q^{l}} .
$$

Thus, the infinitesimal criterion for the invariance of (1) under the supergroup $H$ may be expressed as

$$
\left.\operatorname{pr}^{(k)} \mathrm{V} \Delta_{\nu}\right|_{\Delta_{\nu}=0}=0,
$$

where the $k=\left(k_{1} ; k_{2}\right)$-th superprolongation of the vector field $(7)$ is given by

$$
p r^{(k)} \mathrm{V}=\mathrm{V}+\sum_{r=1}^{q} \sum_{J} \Phi_{J}^{r} \frac{\partial}{\partial A_{J}^{r}}+\sum_{l=1}^{p} \sum_{K} \Lambda_{K}^{l} \frac{\partial}{\partial Q_{K}^{l}},
$$

where $J=\left(J_{1} ; J_{2}\right)=\left(j_{1}^{1}, \ldots, j_{\alpha}^{1} ; j_{1}^{2}, \ldots, j_{\mu}^{2}\right)$ and $K=\left(K_{1} ; K_{2}\right)=\left(k_{1}^{1}, \ldots, k_{\beta}^{1}\right.$;

$\left.k_{1}^{2}, \ldots, k_{\nu}^{2}\right) ; 1 \leq j_{\alpha}^{1}, j_{\mu}^{2}, k_{\beta}^{1}, k_{\nu}^{2} \leq m+n$ are the multi-indices notations for differentiations with respect to the $x_{i}^{\prime} s$ and $\theta_{j}^{\prime} s$. The coefficients $\Phi_{J}^{r}$ and $\Lambda_{K}^{l}$ are given by

$$
\Phi_{J}^{r}=D_{J}\left(\Phi^{r}-\sum_{i=1}^{m} \Xi^{i} A_{i}^{r}-\sum_{j=1}^{n} \Gamma^{j} A_{j}^{r}\right)+\sum_{i=1}^{m} \Xi^{i} A_{J, i}^{r}+\sum_{j=1}^{n} \Gamma^{j} A_{J, j}^{r}
$$

and

$$
\Lambda_{K}^{l}=D_{K}\left(\Lambda^{l}-\sum_{i=1}^{m} \Xi^{i} Q_{i}^{l}-\sum_{j=1}^{n} \Gamma^{j} Q_{j}^{l}\right)+\sum_{i=1}^{m} \Xi^{i} Q_{K, i}^{l}+\sum_{j=1}^{n} \Gamma^{j} Q_{K, j}^{l},
$$

where $A_{i}^{r}=\partial A^{r} / \partial x_{i}, A_{j}^{r}=\partial A^{r} / \partial \theta_{j}, Q_{i}^{l}=\partial Q^{l} / \partial x_{i}$ and $Q_{j}^{l}=\partial Q^{l} / \partial \theta_{j}$.

While differentiating with respect to odd variables, the order of differentiation is very important. We adopt the following notation: for $1 \leq j_{1}, j_{2} \leq n, A_{j_{1}, j_{2}}^{r}$ means that we first differentiate $A^{r}$ with respect to $\theta_{j_{1}}$ then with respect to $\theta_{j_{2}}$. The differentiation of the product of homogeneous Grassmann functions, $F$ and $G$, with respect to odd variables satisfies the rule

$$
\frac{\partial}{\partial \theta}(F G)=\frac{\partial F}{\partial \theta} G+(-1)^{|F|} F \frac{\partial G}{\partial \theta}
$$

where $\frac{\partial}{\partial \theta}$ is understood as the left derivative of $\theta[16]$ and $|F|$ is the parity of $F$ defined by

$$
|F|= \begin{cases}0 & \text { if } \mathrm{F} \text { is even } \\ 1 & \text { if } \mathrm{F} \text { is odd }\end{cases}
$$


After eliminating all dependencies among the derivatives of $A^{r}$ and $Q^{l}$ (since equation (1) must be satisfied), the coefficients (10), (11) and equation (8) will involve expressions with partial derivatives of $\Xi^{i}, \Gamma^{j}, \Phi^{r}$ and $\Lambda^{l}$ with respect to both even and odd dependent and independent variables. Once we evaluate (8) and identify all the coefficients of the different combinations of even and odd dependent variables to zero, we obtain a large number of equations expressed in terms of $\Xi^{i}, \Gamma^{j}, \Phi^{r}$ and $\Lambda^{l}$ and their derivatives with respect to the $x_{i}$ 's and $\theta_{j}$ 's. These are the determining superequations. The resolution of such equations leads to a vector field (7) that is a linear combination of vector fields generating the Lie superalgebra $\mathrm{H}$.

\section{The TB supersymmetric equation}

Let us recall that the classical dispersiveless long wave equations

$$
J_{t}^{1}=-J^{1}\left(J^{1}\right)^{\prime}-\left(J^{2}\right)^{\prime}, J_{t}^{2}=-\left(J^{1} J^{2}\right)^{\prime}
$$

have a number of dispersive generalizations [18, 19, 20]. The study of the integrability of the dispersive generalization of the long water wave equation, also called the two-boson system (TB), has been done [21, 22]. In this paper we shall be concerned with the supersymmetric version of the following system

$$
\begin{aligned}
& J_{t}^{1}=\left(\left(J^{1}\right)^{2}+2 J^{2}-\left(J^{1}\right)^{\prime}\right)^{\prime}, \\
& J_{t}^{2}=\left(2 J^{1} J^{2}+\alpha\left(J^{2}\right)^{\prime}\right)^{\prime},
\end{aligned}
$$

where $J^{1}(x, t)$ and $J^{2}(x, t)$ are respectively the horizontal velocity and the height of the free surface and $\alpha$ is an arbitrary parameter. The prime denotes the derivative with respect to $x$.

The system (15) is integrable and has a trihamiltonian structure [20]. For different values of $\alpha$, the above system could be reduced to many well-known equations such that the non-linear Schrödinger and Burgers equations.

In what follows, $\alpha$ is chosen to be equal to 1 . The most general supersymmetric extension of the dynamical equations (15) which is integrable was introduced by Brunelli and Das [23] and is written as follows

$$
\begin{aligned}
& Q_{t}^{1}=-\left(D^{4} Q^{1}\right)+D\left(D Q^{1}\right)^{2}+2\left(D^{2} Q^{2}\right), \\
& Q_{t}^{2}=\left(D^{4} Q^{2}\right)+2 D^{2}\left(\left(D Q^{1}\right) Q^{2}\right),
\end{aligned}
$$

where $D=\frac{\partial}{\partial \theta}+\theta \frac{\partial}{\partial x}$ is the supercovariant derivative and $Q^{1}, Q^{2}$ are fermionic fields. Replacing $D$ by its value, we obtain

$$
\begin{aligned}
& Q_{t}^{1}=-\left(Q^{1}\right)^{\prime \prime}+2\left(Q^{1}\right)^{\prime} Q_{\theta}^{1}+2\left(Q^{2}\right)^{\prime} \\
& Q_{t}^{2}=\left(Q^{2}\right)^{\prime \prime}+2\left(Q_{\theta}^{1}\right)^{\prime} Q^{2}+2 Q_{\theta}^{1}\left(Q^{2}\right)^{\prime}+2 \theta\left(Q^{1}\right)^{\prime \prime} Q^{2}+2 \theta\left(Q^{1}\right)^{\prime}\left(Q^{2}\right)^{\prime}
\end{aligned}
$$

The fermionic fields $Q^{1}$ and $Q^{2}$ may be expanded as

$$
Q^{1}(x, t, \theta)=\psi^{1}(x, t)+\theta J^{1}(x, t) \text { and } Q^{2}(x, t, \theta)=\psi^{2}(x, t)+\theta J^{2}(x, t),
$$

where $\psi^{1}, \psi^{2}$ are anticommuting functions and $J^{1}, J^{2}$ are commuting ones. Therefore, the supersystem (17) becomes

$$
\begin{aligned}
& J_{t}^{1}=\left(2 J^{2}+\left(J^{1}\right)^{2}-\left(J^{1}\right)^{\prime}\right)^{\prime}, \\
& \psi_{t}^{1}=2\left(\psi^{2}\right)^{\prime}+2\left(\psi^{1}\right)^{\prime} J^{1}-\left(\psi^{1}\right)^{\prime \prime}, \\
& J_{t}^{2}=\left(2 J^{1} J^{2}+\left(J^{2}\right)^{\prime}+2\left(\psi^{1}\right)^{\prime} \psi^{2}\right)^{\prime}, \\
& \psi_{t}^{2}=\left(2 \psi^{2} J^{1}+\left(\psi^{2}\right)^{\prime}\right)^{\prime} .
\end{aligned}
$$


In the bosonic limit, we see that the supersystem (18) reduces to eq. (15) for the particular case $\alpha=1$.

The supersystem (17) is of second order having two even $(x, t)$ and one odd $\theta$ independent variables and the search for its invariance superalgebra will be the object of the next section.

\section{Lie superalgebras of the TB superequations}

Dealing with even and odd variables means that the order in the products must be respected. Furthermore, differentiating Grassmann functions with respect to odd variables may change the type of the resulting function. All these considerations and others were modeled in a package named Grassmann, written in MAPLE language and introduced by Cheb-Terrab [24].

The symmetry superalgebra has been obtained from equations (17) using the package GLie [25]. It helped us to perform the determining superequations (see Appendix A). Solving these superequations, we got the following general solution for the infinitesimals

$$
\begin{aligned}
& \Xi^{1}=c_{1}+2 c_{3} x-2 c_{2} t-\alpha_{1} \& * \theta \\
& \Xi^{2}=c_{4}+4 c_{3} t \\
& \Gamma^{1}=\alpha_{1}+c_{3} \& * \theta \\
& \Lambda^{1}=\alpha_{2}+c_{2} \& * \theta-c_{3} \& * Q^{1} \\
& \Lambda^{2}=-3 c_{3} \& * Q^{2}
\end{aligned}
$$

where $c_{i}, i=1, \ldots, 4$ are even constants and $\alpha_{j}, j=1 \ldots 2$ are odd ones. The symbol " $\&$ *" is the Maple noncommutative multiplication operator.

The supersymmetric two bosons equations (17) admit a (4|2)-dimensional superalgebra generated by:

$$
\begin{aligned}
\mathcal{C}_{1} & =\partial_{x}, \mathcal{C}_{2}=\partial_{t}, \mathcal{C}_{3}=-2 t \partial_{x}+\theta \partial_{Q^{1}} \\
\mathcal{C}_{4} & =2 x \partial_{x}+4 t \partial_{t}+\theta \partial_{\theta}-Q^{1} \partial_{Q^{1}}-3 Q^{2} \partial_{Q^{2}} \\
\mathcal{A}_{1} & =\partial_{\theta}-\theta \partial_{x}, \mathcal{A}_{2}=\partial_{Q^{1}}
\end{aligned}
$$

The supercommutator table of the Lie superalgebra is as follows:

\begin{tabular}{c|cccccc} 
& $\mathcal{C}_{1}$ & $\mathcal{C}_{2}$ & $\mathcal{C}_{3}$ & $\mathcal{C}_{4}$ & $\mathcal{A}_{1}$ & $\mathcal{A}_{2}$ \\
\hline $\mathcal{C}_{1}$ & 0 & 0 & 0 & $2 \mathcal{C}_{1}$ & 0 & 0 \\
$\mathcal{C}_{2}$ & 0 & 0 & $-2 \mathcal{C}_{1}$ & $4 \mathcal{C}_{2}$ & 0 & 0 \\
$\mathcal{C}_{3}$ & 0 & $2 \mathcal{C}_{1}$ & 0 & $-2 \mathcal{C}_{3}$ & $-\mathcal{A}_{1}$ & 0 \\
$\mathcal{C}_{4}$ & $-2 \mathcal{C}_{1}$ & $-4 \mathcal{C}_{2}$ & $2 \mathcal{C}_{3}$ & 0 & $-\mathcal{A}_{1}$ & $\mathcal{A}_{2}$ \\
$\mathcal{A}_{1}$ & 0 & 0 & $\mathcal{A}_{1}$ & $\mathcal{A}_{1}$ & $-2 \mathcal{C}_{1}$ & 0 \\
$\mathcal{A}_{2}$ & 0 & 0 & 0 & $-\mathcal{A}_{2}$ & 0 & 0
\end{tabular}

As usual, commutation relations are satisfied for even-even and even-odd products while anticommutation relations are satisfied for odd-odd products.

Therefore, the one-parameter supergroups of transformations $\mathcal{G}_{i}$ generated by the $\left\{\mathcal{C}_{i}, \mathcal{A}_{j}\right\}$ are given as follows:

$$
\begin{array}{ll}
\mathcal{G}_{1}:\left(x+\epsilon, t ; \theta, Q^{1}, Q^{2}\right), & \mathcal{G}_{2}:\left(x, t+\epsilon ; \theta, Q^{1}, Q^{2}\right), \\
\mathcal{G}_{3}:\left(-2 t \epsilon+x, t ; \theta, Q^{1}+\theta \epsilon Q^{2}\right), & \mathcal{G}_{4}:\left(x e^{2 \epsilon}, t e^{4 \epsilon} ; \theta e^{\epsilon}, Q^{1} e^{-\epsilon}, Q^{2} e^{-3 \epsilon}\right), \\
\mathcal{G}_{5}:\left(x-\epsilon \theta, t ; \theta+\epsilon, Q^{1}, Q^{2}\right), & \mathcal{G}_{6}:\left(x, t ; \theta, Q^{1}+\epsilon, Q^{2}\right)
\end{array}
$$




\section{Translationally invariant solutions}

In this section, we shall consider some solutions of the TB superequations which are invariant under subgroups involving the generator $\lambda \mathcal{C}_{1}+\mathcal{C}_{2}$. The new independent variable, $y=x-\lambda t$, is called the invariant and the supersystem (17) becomes, in terms of the new variables $(\theta, y)$,

$$
\begin{aligned}
& \lambda Q_{y}^{1}-Q_{y y}^{1}+2 Q_{y}^{1} Q_{\theta}^{1}+2 Q_{y}^{2}=0, \\
& \lambda Q_{y}^{2}+Q_{y y}^{2}+2 Q_{\theta y}^{1} Q^{2}+2 Q_{\theta}^{1} Q_{y}^{2}+2 \theta Q_{y y}^{1} Q^{2}+2 \theta Q_{y}^{1} Q_{y}^{2}=0 .
\end{aligned}
$$

Now, expressing $Q^{1}$ and $Q^{2}$ in components as:

$$
Q^{1}=\psi^{1}(y)+\theta J^{1}(y), Q^{2}=\psi^{2}(y)+\theta J^{2}(y),
$$

we get the following nonlinear coupled supersystem of ordinary differential equations:

$$
\begin{aligned}
\frac{1}{2}\left(J_{y}^{1}-\lambda J^{1}-\left(J^{1}\right)^{2}-c_{1}\right) & =J^{2} \\
\psi_{y y}^{1}-\left(\lambda+2 J^{1}\right) \psi_{y}^{1}-2 \psi_{y}^{2} & =0 \\
J_{y}^{2}+\left(\lambda+2 J^{1}\right) J^{2}+2 \psi_{y}^{1} \psi^{2} & =c_{2}, \\
\psi_{y}^{2}+\left(\lambda+2 J^{1}\right) \psi^{2} & =\mu_{1},
\end{aligned}
$$

where the letters $c_{i}$ and $\mu_{j}$ are considered respectively even and odd constants of integration.

In order to solve it, we first put $J^{2}$ as given by eq. (26) into eq. (28). We then get

$$
J_{y y}^{1}-2\left(J^{1}\right)^{3}-3 \lambda\left(J^{1}\right)^{2}-\left(\lambda^{2}+2 c_{1}\right) J^{1}+4 \psi_{y}^{1} \psi^{2}=\lambda c_{1}+2 c_{2} .
$$

Next, we can take the new function $J(y)=J^{1}(y)+\frac{\lambda}{2}$ to write the system of eqs. (27), (29) and (30) in the form:

$$
\begin{aligned}
\psi_{y y}^{1}-2 J \psi_{y}^{1}-2 \psi_{y}^{2} & =0, \\
\psi_{y}^{2}+2 J \psi^{2} & =\mu_{1}, \\
J_{y y}-2(J)^{3}-\omega^{2} J+4 \psi_{y}^{1} \psi^{2} & =2 c_{2},
\end{aligned}
$$

where $\omega=\sqrt{2 c_{1}-\lambda^{2} / 2}$. The equations (31) and (32) are linear in $\psi^{1}$ and $\psi^{2}$ and give rise to the following expressions for $\psi^{2}$ and $\psi^{1}$ respectively, in terms of the unknown function $J$ :

$$
\psi^{2}(y)=\left(\mu_{1} \int e^{2 \int J(y) d y} d y+\mu_{2}\right) e^{-2 \int J(y) d y}
$$

and

$$
\psi^{1}(y)=\int \rho(y) d y+\mu_{4}
$$

with

$$
\rho(y)=\left(2 \int\left(\mu_{1}-2 J(y) \psi^{2}(y)\right) e^{-2 \int J(y) d y} d y+\mu_{3}\right) e^{2 \int J(y) d y} .
$$

Note that $\mu_{2}, \mu_{3}$ and $\mu_{4}$ are new odd integration constants. The equation (33) is not easy to integrate but some solutions may be given. For example, if we choose the function $J(y)$ on the form

$$
J(y)=\frac{a}{y},
$$


where $a$ is a constant, the explicit forms of $\psi^{2}$ and $\psi^{1}$ are easily deduced. Indeed we get

$$
\psi^{2}(y)=\frac{\mu_{1}}{1+2 a} y+\mu_{2} y^{-2 a},
$$

and

$$
\psi^{1}(y)=\frac{\mu_{1}}{1-4 a^{2}} y^{2}+\frac{\mu_{2}}{1-2 a} y^{1-2 a}+\frac{\mu_{3}}{1+2 a} y^{2 a+1}+\mu_{4} .
$$

These solutions are consistent only for $a=-1$ or $a=1$ since we have to take into account the eq. (33). The constants $\mu_{i}, i=1, \ldots, 4$ being odd Grassmann numbers, their square disappear and it remains the following conditions and solutions of the supersystem (26-29), in terms of the original variables $x$ and $t$ for the two cases:

$\underline{\text { For } a=-1 \text {, we get }}$

$$
\begin{aligned}
J^{1}(x, t) & =-\frac{1}{x-\lambda t}-\frac{\lambda}{2} \\
J^{2}(x, t) & =-\frac{\omega^{2}}{4} \\
\psi^{1}(x, t) & =\frac{\mu_{1}}{3}(x-\lambda t)^{2}(x-\lambda t-1)-\frac{\mu_{3}}{x-\lambda t}+\mu_{4}, \\
\psi^{2}(x, t) & =-\frac{\mu_{1}}{x-\lambda t}+\mu_{1}(x-\lambda t)^{2}
\end{aligned}
$$

since we have to impose $\mu_{1}=\mu_{2}$ and the other constants must verify

$$
\omega^{2}+4 \mu_{1} \mu_{3}=0 \text { and } c_{2}=2 \mu_{1} \mu_{3}
$$

For $a=1$, we get

$$
\begin{aligned}
J^{1}(x, t) & =\frac{1}{x-\lambda t}-\frac{\lambda}{2} \\
J^{2}(x, t) & =-\frac{\omega^{2}}{4}-\frac{1}{(x-\lambda t)^{2}}, \\
\psi^{1}(x, t) & =\frac{\mu_{1}}{3}(x-\lambda t)^{2}(x-\lambda t-1)-\frac{\mu_{2}}{x-\lambda t}+\mu_{4}, \\
\psi^{2}(x, t) & =\frac{\mu_{1}}{3}(x-\lambda t)+\frac{\mu_{2}}{(x-\lambda t)^{2}},
\end{aligned}
$$

since we have $\mu_{1}=\mu_{3}$ and the other constants must verify

$$
\omega^{2}+\frac{4}{3} \mu_{1} \mu_{2}=0 \text { and } c_{2}=2 \mu_{1} \mu_{2}
$$

Let us consider the case where the product $\psi_{y}^{1}(y) \psi^{2}(y)$ is a constant in eq. (33). Taking into account the eqs. (31) and (32) and the fact that $\psi^{1}$ and $\psi^{2}$ are odd functions, we see that it is realised only when $\psi^{2}=0$. This means that we are considering solutions of the system (26-29) for which we have $\psi^{2}=0$ and $\mu_{1}=0$. The equation (33) is now the modified KdV equation for $J(y)$ and some particular solutions may be given. Indeed, we know that it admits the soliton solution

$$
J(y)=\frac{i \omega}{\cosh \omega y}
$$


We can then write the solution $J^{1}, J^{2}$ and $\psi^{1}$ as functions of $x$ and $t$ :

$$
\begin{aligned}
J^{1}(x, t) & =-\frac{\lambda}{2}+\frac{i \omega}{\cosh (\omega(x-\lambda t))} \\
J^{2}(x, t) & =\frac{\omega^{2}}{2}\left\{\left(-\frac{1}{2}+\frac{1}{\cosh ^{2}(\omega(x-\lambda t))}\right)-i \frac{\sinh (\omega(x-\lambda t))}{\cosh ^{2}(\omega(x-\lambda t))}\right\} \\
\text { and } & \\
\psi^{1}(x, t) & =\mu_{3} f(x, t)+\mu_{4}
\end{aligned}
$$

with

$$
f(x, t)=-(x-\lambda t)+\frac{2}{\omega}\left\{\tanh (\omega(x-\lambda t))-i\left(1+\frac{1}{\cosh (\omega(x-\lambda t))}\right)\right\} .
$$

Finally, we can also consider the rational solution

$$
J(y)=a+\frac{4 a}{4 a y^{2}-1}
$$

of eq. (33) where $\omega^{2}=-6 a^{2}$ and $c_{2}=2 a^{3}$. It leads to

$$
\begin{aligned}
J^{1}(x, t) & =-\frac{\lambda}{2}+a+\frac{4 a}{4 a^{2}(x-\lambda t)^{2}-1}, \\
J^{2}(x, t) & =a^{2}-\frac{4 a^{2}}{4 a^{2}(x-\lambda t)^{2}-1}-\frac{8 a^{2}}{\left(4 a^{2}(x-\lambda t)^{2}-1\right)^{2}}, \\
\text { and } & =\mu_{3}\left(\frac{e^{2 a(x-\lambda t)}}{2 a}-2 \frac{e^{2 a(x-\lambda t)}}{a(2 a(x-\lambda t)+1)}\right)+\mu_{4} .
\end{aligned}
$$

\section{Conclusion}

Through this example of the supersymmetric TB equations, we have shown that it is possible to solve Grassmann-valued differential equations by the method of symmetries. Computer calculations has greatly helped us in carrying out the symmetry algebra. In a future work, we will intend to apply the method of conditionnal symmetries [26] to such supersystems in order to obtain other interesting class of solutions. Also the problem of solving such supersystems analytically or numerically with initial conditions could be addressed.

\section{Acknowledgements}

The research of V. Hussin is partially supported by research grants from NSERC of Canada and FCAR du Gouvernement du Québec. M. I. Ayari thanks the "Fonds FCAR du Gouvernement du Québec" for a scholarship.

\section{Appendix}




\section{A Computation of the determining superequations for the TB equations}

Here we give the $4 t h$ run execution of the input file and the Maple session for the supersymmetric two bosons equations. It is important to mention that the code GLie compute only the determining superequations for any high order SGVDE. Their resolution should be done by hand. In an interactive way, we run the program and explore the dependency of the infinitesimals. Then we feed back the results into the input file. We repeat this procedure until we get the simplest supersystem. For more description and use of this package see [25].

The Maple CPU Time is about 248.5 seconds. Note that "\&*" is the Maple noncommutative multiplication operator and "gdiff" is an extension of the Maple conventional differentiation operator "diff" introduced by Cheb-Terrab [24] to fill the gap between the standard resources of Maple and our needs. This operator respects the Leibniz rule (12) and the correct form of chain rule of higher order differentiations.

\section{The Input File}

The input data for GLie must have the following arrays introduced in an ascii file. Note that DifDependOVar contains all the partial differential terms for odd dependent variables that figure in the supersystem.

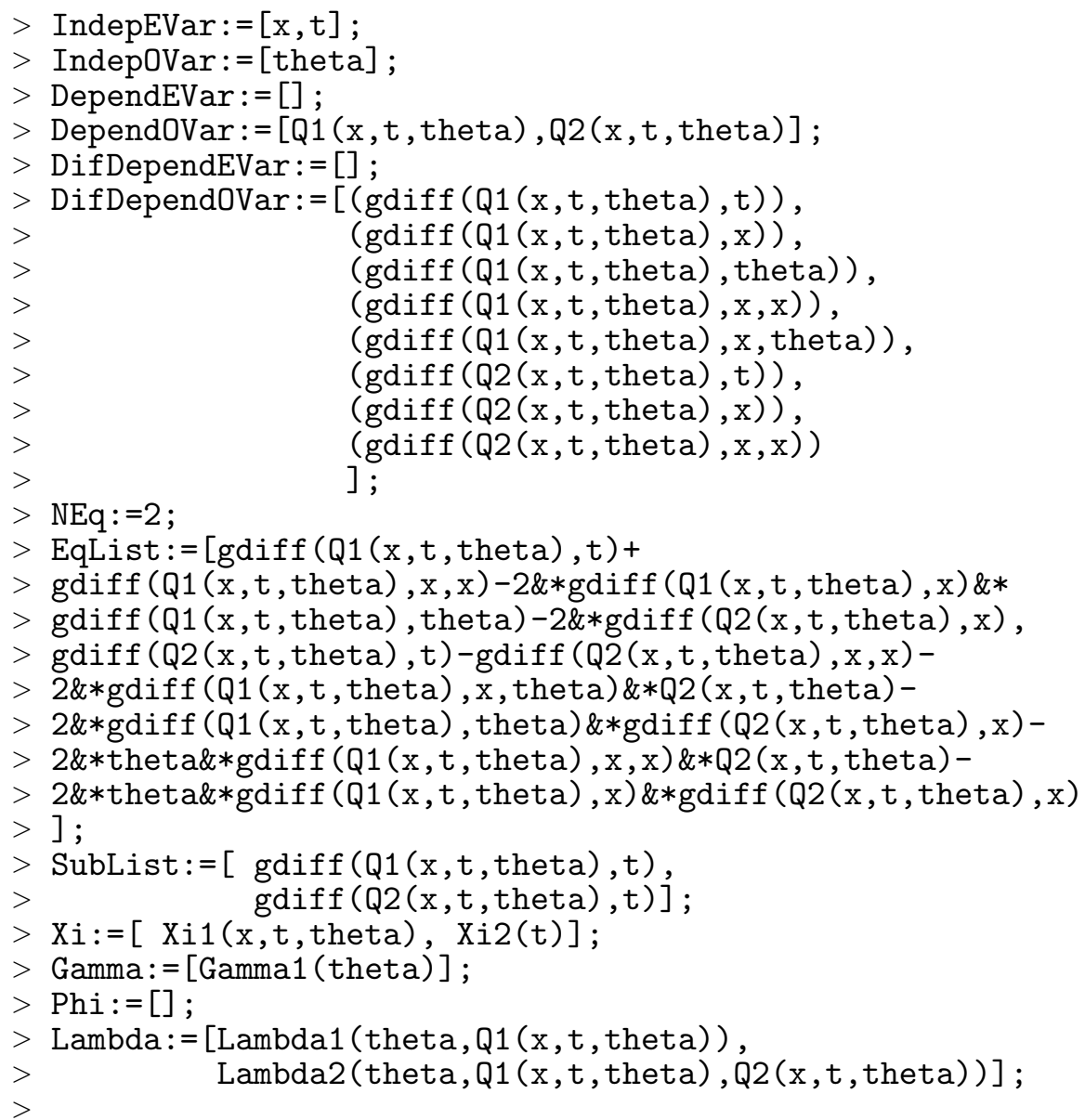

\section{The Output File}

Using the tools of the Maple session, the results could be exported in $\mathrm{AT}_{\mathrm{E}} \mathrm{X}$ form. Following are the determining superequation. Their resolution has lead to the infinitesimals (19)-(23). 


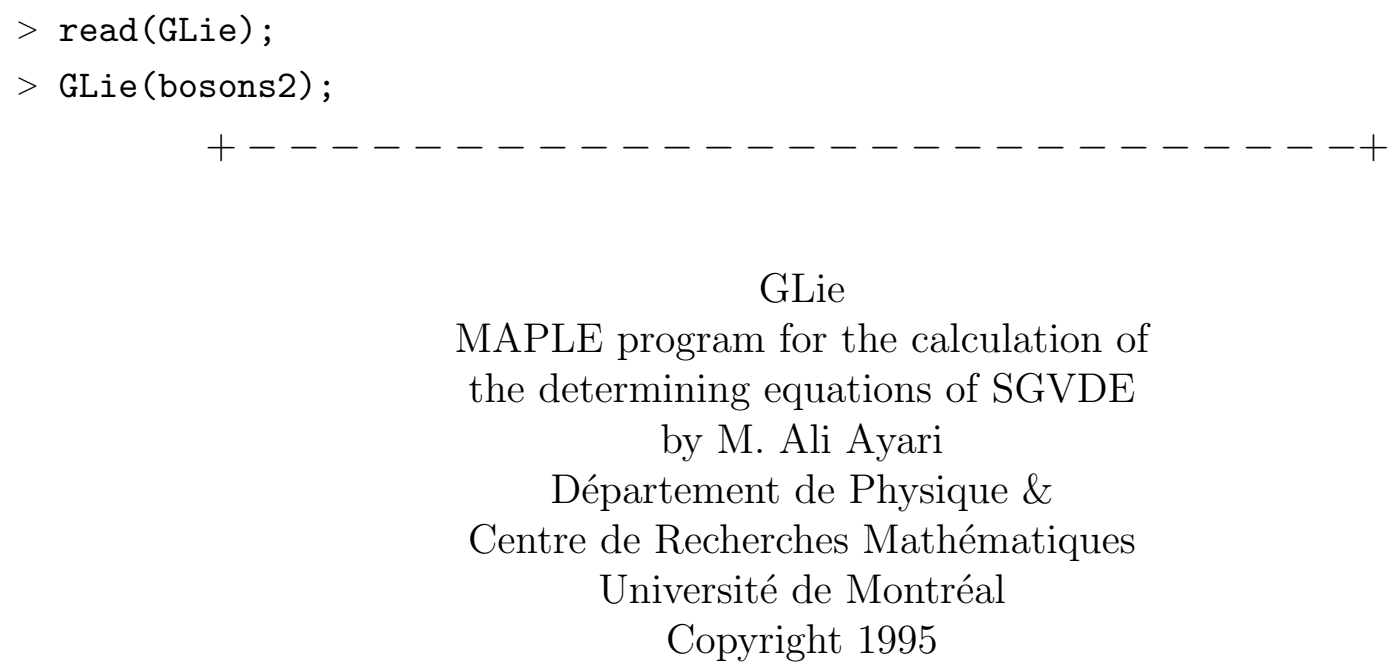

GLie

MAPLE program for the calculation of the determining equations of SGVDE

by M. Ali Ayari

Département de Physique \&

Centre de Recherches Mathématiques

Université de Montréal

Copyright 1995

Independent Even Variables....total of 2

$$
[x, t]
$$

Independent Odd Variables...total of 1

$[\theta]$

Dependent Even Variables...total of 0

Dependent Odd Variables....total of 2

$$
[\mathrm{Q} 1(x, t, \theta), \mathrm{Q} 2(x, t, \theta)]
$$

Number of Equations............total of 2

Equations List..............

$$
\begin{aligned}
& D_{2}(Q 1)(x, t, \theta)+D_{1,1}(Q 1)(x, t, \theta) \\
&-\left(\left(2 \& * D_{1}(Q 1)(x, t, \theta)\right) \& * D_{3}(Q 1)(x, t, \theta)\right) \\
&-\left(2 \& * D_{1}(Q 2)(x, t, \theta)\right)=0 \\
& D_{2}(Q 2)(x, t, \theta)-D_{1,1}(Q 2)(x, t, \theta) \\
&-\left(\left(2 \& * D_{1,3}(Q 1)(x, t, \theta)\right) \& * \mathrm{Q} 2(x, t, \theta)\right) \\
&-\left(\left(2 \& * D_{3}(Q 1)(x, t, \theta)\right) \& * D_{1}(Q 2)(x, t, \theta)\right) \\
&-\left(\left((2 \& * \theta) \& * D_{1,1}(Q 1)(x, t, \theta)\right) \& * \mathrm{Q} 2(x, t, \theta)\right) \\
&-\left(\left((2 \& * \theta) \& * D_{1}(Q 1)(x, t, \theta)\right) \& * D_{1}(Q 2)(x, t, \theta)\right)=0
\end{aligned}
$$


Substitution List............

$$
\begin{aligned}
& D_{2}(Q 1)(x, t, \theta) \\
& D_{2}(Q 2)(x, t, \theta)
\end{aligned}
$$

The Determining Superequations

Total number of determining superequations : 9

.......Equation No: 1

$$
\begin{aligned}
& -2 D_{2}(\Lambda 1)(\theta, \mathrm{Q} 1(x, t, \theta))-2 \mathrm{D}(\Xi 2)(t)+2 D_{1}(\Xi 1)(x, t, \theta) \\
& \quad+2 \mathrm{D}(\Gamma 1)(\theta)=0
\end{aligned}
$$

......Equation No: 2

$$
\begin{aligned}
& 2 D_{2}(\Lambda 1)(\theta, \mathrm{Q} 1(x, t, \theta))-2 \mathrm{D}(\Xi 2)(t) \\
& \quad-2 D_{3}(\Lambda 2)(\theta, \mathrm{Q} 1(x, t, \theta), \mathrm{Q} 2(x, t, \theta))+2 D_{1}(\Xi 1)(x, t, \theta)=0
\end{aligned}
$$

.......Equation No: 3

$$
\begin{aligned}
& -D_{2}(\Xi 1)(x, t, \theta)-2 D_{1}(\Lambda 1)(\theta, \mathrm{Q} 1(x, t, \theta))-D_{1,1}(\Xi 1)(x, t, \theta) \\
& -2 D_{2}(\Lambda 2)(\theta, \mathrm{Q} 1(x, t, \theta), \mathrm{Q} 2(x, t, \theta))=0
\end{aligned}
$$

.......Equation No: 4

$$
\mathrm{D}(\Xi 2)(t)-2 D_{1}(\Xi 1)(x, t, \theta)=0
$$

.......Equation No: $\quad 5$

$$
\begin{aligned}
-2 D_{2} & (\Lambda 2)(\theta, \mathrm{Q} 1(x, t, \theta), \mathrm{Q} 2(x, t, \theta)) \\
& +2(\theta \& * \Lambda 2(\theta, \mathrm{Q} 1(x, t, \theta), \mathrm{Q} 2(x, t, \theta))) \\
& -4 \& *\left(D_{1}(\Xi 1)(x, t, \theta), \theta, \mathrm{Q} 2(x, t, \theta)\right) \\
& +2 \& *\left(D_{2}(\Lambda 1)(\theta, \mathrm{Q} 1(x, t, \theta)), \theta, \mathrm{Q} 2(x, t, \theta)\right) \\
& +2 \mathrm{D}(\Xi 2)(t)(\theta \& * \mathrm{Q} 2(x, t, \theta)) \\
& +2\left(\mathrm{Q} 2(x, t, \theta) \& * D_{3}(\Xi 1)(x, t, \theta)\right) \\
& -2 \& *\left(D_{3}(\Lambda 2)(\theta, \mathrm{Q} 1(x, t, \theta), \mathrm{Q} 2(x, t, \theta)), \theta, \mathrm{Q} 2(x, t, \theta)\right) \\
& -2(\mathrm{Q} 2(x, t, \theta) \& * \Gamma 1(\theta))=0
\end{aligned}
$$

.Equation No: 6

$$
\begin{aligned}
-2 & \Lambda 2(\theta, \mathrm{Q} 1(x, t, \theta), \mathrm{Q} 2(x, t, \theta)) \\
& +2\left(D_{3}(\Lambda 2)(\theta, \mathrm{Q} 1(x, t, \theta), \mathrm{Q} 2(x, t, \theta)) \& * \mathrm{Q} 2(x, t, \theta)\right) \\
& -2 \mathrm{D}(\Xi 2)(t) \mathrm{Q} 2(x, t, \theta) \\
& -2\left(D_{2}(\Lambda 1)(\theta, \mathrm{Q} 1(x, t, \theta)) \& * \mathrm{Q} 2(x, t, \theta)\right) \\
& +2\left(D_{1}(\Xi 1)(x, t, \theta) \& * \mathrm{Q} 2(x, t, \theta)\right) \\
& +2(\mathrm{D}(\Gamma 1)(\theta) \& * \mathrm{Q} 2(x, t, \theta))=0
\end{aligned}
$$


.......Equation No: 7

$$
\begin{aligned}
& -2 \& *\left(D_{1,1}(\Xi 1)(x, t, \theta), \theta, \mathrm{Q} 2(x, t, \theta)\right) \\
& \quad+2\left(\mathrm{Q} 2(x, t, \theta) \& * D_{1,2}(\Lambda 1)(\theta, \mathrm{Q} 1(x, t, \theta))\right) \\
& \quad+2\left(\mathrm{Q} 2(x, t, \theta) \& * D_{1,3}(\Xi 1)(x, t, \theta)\right)=0
\end{aligned}
$$

.......Equation No: 8

$$
\begin{aligned}
& D_{1,1}(\Xi 1)(x, t, \theta)-2 D_{1}(\Lambda 1)(\theta, \mathrm{Q} 1(x, t, \theta))-D_{2}(\Xi 1)(x, t, \theta) \\
& \quad+2 D_{2}(\Lambda 2)(\theta, \mathrm{Q} 1(x, t, \theta), \mathrm{Q} 2(x, t, \theta))=0
\end{aligned}
$$

........Equation No: 9

$$
\begin{gathered}
-2 D_{2,3}(\Lambda 2)(\theta, \mathrm{Q} 1(x, t, \theta), \mathrm{Q} 2(x, t, \theta))+2 D_{3}(\Xi 1)(x, t, \theta) \\
\quad-2\left(D_{2}(\Lambda 1)(\theta, \mathrm{Q} 1(x, t, \theta)) \& * \theta\right)-2 \Gamma 1(\theta) \\
+4\left(D_{1}(\Xi 1)(x, t, \theta) \& * \theta\right)-2 \mathrm{D}(\Xi 2)(t) \theta=0
\end{gathered}
$$

\section{References}

[1] Yu I. Manin and A. O. Radul, A Supersymmetric Extension of the Kadomtsev-Petviashvili Hierarchy, Commun. Math. Phys. 98, (1985) 65-77.

[2] C. Elphick, Classes of Exactly Solvable Nonlinear Evolution Equations for Grassmann Variables: The Normal Form Method, J. Math. Phys. 28, (1987) 1243-1249.

[3] P. Mathieu, Supersymmetric extension of the Korteweg-de Vries equation, J. Math. Phys. 29, (1988) 2499-2506.

[4] B. W. Fatyga, V. A. Kostelecký and D. R. Truax, Grassmann-Valued Fluid Dynamics, J. Math. Phys. 30, (1989) 1464-1472.

[5] P. Labelle and P. Mathieu, A New $N=2$ Supersymmetric Korteweg-de Vries Equation, J. Math. Phys. 32, (1991), 923-927.

[6] G. H. M. Roelofs and P. H. M. Kersten, Supersymmetric Extension of The Nonlinear Schrödinger Equation: Symmetries and Coverings, J. Math. Phys. 33, (1992) 2185-2206.

[7] C. M. Yung, The $N=2$ supersymmetric Boussinesq hierarchies, Phys. Lett. B 309, (1993) 75-84.

[8] S. Belluci, E. Ivanov, S. Krivonos and A. Pichugin, $N=2$ super Boussinesq hierarchy: Lax pairs and conservation laws, Phys. Lett. B 312, (1993) 463-470.

[9] A. Ibort, A. L. Martinez and R. Medina, Explicit solutions of supersymmetric KP hierarchies: supersolitons and solitinos, J. Math. Phys. 37, (1996) 6157-6172.

[10] R. Cianci, Superespace first-order partial differential equations through the cartan-Kähler integration theorem, J. Math. Phys. 29, (10) (1988) 2156-2161. 
[11] M. A. Ayari, Supergroupes de Lie et solutions invariantes pour des équations différentielles non-linéaires à valeurs de Grassmann, Ph.D. Dissertation, Université de Montréal (1997).

[12] M. A. Ayari and V. Hussin, Computation of Lie Supersymmetries for Grassmann-Valued Differential Equations, Proceedings of The XXI. International Colloquium on Group Theoretical Methods in Physics, 15-20 July 1996, Goslar, Germany.

[13] M. A. Ayari, V. Hussin and P. Winterntiz, Lie Supergoups and Explicit solutions for the Super $\mathrm{KdV}$ equations $(\mathrm{N}=2)$, (1998) (in preparation).

[14] P. J. Olver Applications of Lie Groups to Differential Equations, (Springer-Verlag, 1986).

[15] G.W. Bluman and S. Kumei, Symmetries and Differential Equations, (New York: Springer, 1989).

[16] F.A. Berezin, Introduction to Superanalysis, (Dortrecht, Holland: Reidel, 1987).

[17] B. Kostant, Graded Manifolds, Graded Lie Theory, and prequantization, Proceedings Differential Geometrical Methods in Mathematical Physics (Bonn, 1975), Lecture Notes in Mathematics 570, (1977) 177-307.

[18] L. J. F. Broer, Approximate equations for long water waves, Appl. Sci. Res. 31, (1975) 377-395.

[19] D. J. Kaup, A higher-order water-wave equation and the method for solving it, Prog. Theor. Phys. 54, (1975) 396-408.

[20] B. A. Kupershmidt, Mathematics of dispersive water waves, Commun. Math. Phys. 99, (1985) $51-73$.

[21] H. Aratyn, L. A. Fereirra, J. F. Gomes and A. H. Zimerman, On $W_{\infty}$ Algebras, Gauge Equivalence of KP Hierarchies, Two-Boson realizations and their KdV Reductions, Nucl. Phys. B 402, (1993) 85.

[22] L. Bonora and C. S. Xiong, An alternative approach to KP hierarchy in matrix models. Phys. Lett. B 285, (1992) 191-198.

[23] J. C. Brunelli and A. Das, The supersymmetric two bosons hierarchies, Phys. Lett. B 337 , (1994) 303-307.

[24] E. S. Cheb-Terrab, Symbolic Computing with Anticommutative and Noncommutative Variables, accepted for publication, MapleTech/January 1998; http://dft.if.uerj.br/symbcomp.htm.

[25] M. A. Ayari and V. Hussin, GLie: A MAPLE Program for Lie Supersymmetries of Grassmannvalued Differential Equations, Comp. Phys. Comm. 100, (1997) 157-176.

[26] M. I. Ayari and A. M. Grundland, Conditional Symmetries for kth Order Partial Differential Equations, submitted to J. Math. Anal. Appl. 28 pages (1997); Preprint CRM-2488, August 1997, Université de Montréal. 